
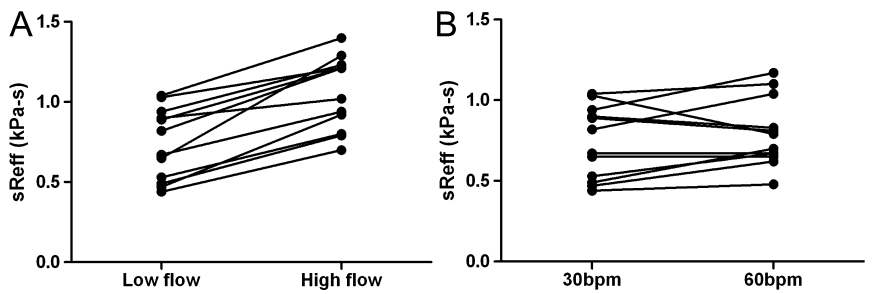

Abstract P126 Figure 1 Within-subject changes in sReff when $(A)$ flows are doubled but breathing frequency remains constant at $30 \mathrm{bpm}$, and (B) when flows remain relatively constant but breathing frequency is doubled.

Conclusion The true impact of breathing pattern on sRaw relates more to flows attained than to breathing frequency. To facilitate improved repeatability and minimise inter-subject and inter-centre variability, it is essential that subjects are encouraged to breathe quietly and naturally during plethysmographic recordings of sRaw. Software adaptations that allow accurate display of flows during data collection and analysis are required before guidelines pertaining to flow can be generated.

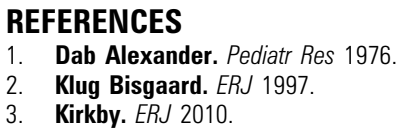

\section{P127 DOES IT MATTER HOW THEY BREATHE?-PERCEPTIONS IN COPD PATIENTS WHEN ADOPTING DIFFERENT ROUTES OF BREATHING}

doi:10.1136/thx.2010.150987.28

${ }^{1} \mathrm{~A} J$ Williams, ${ }^{1} \mathrm{~A}$ Pitcher, ${ }^{2} \mathrm{P}$ Thomas, ${ }^{2} \mathrm{R}$ Baker. ${ }^{1}$ Department of Thoracic Medicine, Royal Bournemouth Hospital, Bournemouth, UK; ${ }^{2}$ Dorset Research and Development Support Unit, School of Health and Social Care, Bournemouth University, Bournemouth, UK

Introduction We have reported that normal subjects prefer nasal breathing (NB) and that the adoption of mouth breathing $(\mathrm{MB})$ is associated with uncomfortable sensations including breathlessness. ${ }^{1}$ $\mathrm{MB}$ may predispose to the perception of breathlessness by dynamically changing chest wall mechanics (CWM), and thus proprioceptive input. This study has been extended to COPD patients in whom airways resistance and dynamic hyperinflation is likely to alter CWM.

Method 20 COPD patients, mean age 71 years (range 47-89), FEV 1 mean 0.81 (range $0.4-2.02 \mathrm{l}$ ), whilst at rest (tidal breathing), undertook a $2 \times 2$ min cross over exercise during which subjects were requested to note their perceptual experiences when randomly allocated to either NB or, after a break, MB. The results have been compared to the individuals preferred route of breathing normally and 20 normal subjects (controls). ${ }^{1}$

Results $10 / 20$ (50\%) of COPD patients during NB found the exercise to be uncomfortable compared to only $3 / 20$ (15\%) controls $(p=0.04) .9 / 20(45 \%)$ witnessed discomfort with breathing/breathlessness, $0 / 20$ dry mouth and $2 / 20(5 \%)$ a desire to cough. $13 / 20$ (65\%) of COPD patients during $\mathrm{MB}$ found the exercise to be uncomfortable compared to $10 / 20(50 \%)$ controls $(p=0.52) .8 / 20$ (40\%) COPD patients witnessed discomfort with breathing/ breathlessness, and dry mouth 6/20 (30\%). 6/20 (30\%) COPD patients preferred NB, $7 / 20$ (35\%) had no preference and $7 / 20(35 \%)$ preferred $\mathrm{MB}$ during normal breathing in usual life. This compares to $13 / 20(65 \%), 4 / 20(20 \%)$ and $3 / 20(15 \%)$ respectively for controls. $9 / 20(45 \%)$ COPD patients had a positive Nijmegen score $(>23)$ (compared to $0 / 20$ controls) and Hospital Anxiety Depression $(\mathrm{HAD})$ scores were greater in COPD $(p<0.001)$. Depression in
COPD patients was strongly associated with a preference for $M B$ normally $(r=0.6, p=0.007)$.

Conclusions This study has shown that COPD patients, in contrast to controls, have adopted a shift in breathing preferences to favour $\mathrm{MB}$. High scores for Nijmegen and $\mathrm{HAD}$ in COPD suggest ventilatory dysfunction with depression closely linked to $M B$. We hypothesise that $\mathrm{MB}$ in $\mathrm{COPD}$ patients is intricately linked to high levels of ventilatory dysfunction and depression.

\section{REFERENCE}

1. Thorax 2009;64:A153.

\section{P128 THE CHALLENGES OF HYPERTONIC SALINE IN NON-CF BRONCHIECTASIS}

doi:10.1136/thx.2010.150987.29

T M Small, H Groves, P McAlinden, J Davison, A DeSoyza. Freeman Hospital, Newcastle upon Tyne, UK

Background Non-Cystic Fibrosis (CF) bronchiectasis is an understudied clinical area. Many studies are extrapolated from CF despite prior evidence suggesting this is not wholly appropriate. Recent BTS guidelines have suggested a role for Hypertonic Saline (HS) after findings from Kellet et al (2008). In that study, a nebulised (7\%) HS challenge test was done and subjects were to be excluded from ongoing HS therapy if they reported 'chest tightness', 'wheeze' or 'difficulty in breathing', or had a $10 \%$ reduction in spirometry following inhalation of HS. Notably none of 23 patients were excluded on these criterion. We hypothesised that a fall in FEV greater than $10 \%$ would be more common in a non study population but of limited clinical significance.

Methods We reviewed our $6 \%$ hypertonic saline challenge (baseline, post $2.5 \mathrm{mg}$ Salbutamol and post 6\% HS) data from consecutive patients commenced on this therapy due to mucus retention despite other interventions (eg, physiotherapy and/or mucolytic) Results: 33 patients were identified (20F, 13M) mean age $61.5 \pm 13$ years, mean baseline $\mathrm{FEV}_{1}$ was $1.37 \mathrm{~L} \pm 0.7$ (range 0.4-2.65) mean $\mathrm{FEV}_{1} \%$ predicted $54 \% \pm 25 \%$ (range $23-111 \%$ ). One patient's data were excluded due to inability to reproducibly perform spirometry. The mean change in $\mathrm{FEV}_{1}$ from baseline was $+4.3 \% \pm 10.8 \%$ (range -21 to -36 ) and fall from post Salbutamol was $-0.64 \pm 11.6 \%$ (range -14 to $+14 \%)$. Seven patients have stopped HS therapy either due to chest tightness or taste (20\%).

Conclusions This cohort is larger than that previously reported and includes a wider range of airflow obstruction severity. No exclusion criterion were applied to trying HS. The overall fall during an acute HS challenge in an unselected bronchiectasis population is small. Despite this many patients discontinue HS within 3 months of initiation.

\section{P129 LUNG CLEARANCE INDEX (LCI) IS A SENSITIVE MARKER OF EARLY AIRWAY CHANGES IN SMOKERS WITH NORMAL SPIROMETRY}

doi:10.1136/thx.2010.150987.30

${ }^{1} \mathrm{~N}$ J Bell, ${ }^{1} \mathrm{~A}$ R Horsley, ${ }^{1} \mathrm{P}$ A Reid, ${ }^{2} \mathrm{H}$ S Sheridan, ${ }^{3} \mathrm{M}$ Monaghan, ${ }^{3} \mathrm{H}$ Connelly, ${ }^{1} \mathrm{~A} P$ Greening, ${ }^{1} \mathrm{~J}$ A Innes. ${ }^{1}$ Respiratory Unit, Western General Hospital, Edinburgh, UK; ${ }^{2}$ Medical Genetics Section, University of Edinburgh, Edinburgh, UK; ${ }^{3}$ Stop Smoking Services, NHS Lothian, Edinburgh, UK

Introduction and objectives Abnormalities in indices of gas mixing derived from multiple breath nitrogen washouts have been demonstrated in smokers without spirometric evidence of airflow obstruction, which improved on smoking cessation. We report initial data from a study investigating the ability of a simpler 